\title{
EFFECT OF CAPSAICIN ON THE OXIDATIVE STRESS AND DOPAMINE CONTENT IN THE TRANSGENIC DROSOPHILA MODEL OF PARKINSON'S DISEASE
}

\author{
Yasir H. Siddique, ${ }^{*}$ Falaq NaZ and Smita Jyoti \\ Drosophila Transgenic Laboratory, Section of Genetics, Department of Zoology, \\ Aligarh Muslim University, Aligarh, Uttar Pradesh 202002, India
}

(Received: November 14, 2017; accepted: March 8, 2018)

\begin{abstract}
In the present study the effect of capsaicin was studied on PD model flies expressing human alpha synuclein. First the potential of scavenging superoxide anion and free radicals by capsaicin at doses of 20, 40, 80 and $100 \mu \mathrm{M}$ was estimated. The PD flies were allowed to feed separately on the diet containg 20,40 , 80 and $100 \mu \mathrm{M}$ of capsaicin, respectively, for 24 days. After 24 days of exposure, fly head homogenate was prepared from each group and was used to estimate glutathione (GSH), protein carbonyl (PC), dopamine content, lipid peroxidation (LPO), glutathione-S-transferase (GST) and monoamine oxidase (MAO) activity. A dose dependent significant increase in the potential of scavenging superoxide anions and free radicals by capsaicin was observed for the doses of 20,40, 80 and $100 \mu \mathrm{M}$. The exposure of capsaicin not only significantly increased the GSH (max. by 1.37 -fold), and dopamine (max. by 1.56 -fold) content but also reduced LPO (max. by 1.8-fold), GST (max. by 1.26-fold), MAO activities (max. by 1.60 -fold) and PC content (max. by 1.95-fold), compared to unexposed PD flies $(p<0.05)$. The results suggest the protective role of capsaicin against the PD symptoms.
\end{abstract}

Keywords: Capsaicin - Parkinson's disease - reactive oxygen species - oxidative stress

\section{INTRODUCTION}

Parkinson's disease (PD) is a neuropathological disorder involving the degeneration of dopaminergic neurons in the substantia nigra pars compacta of the mid-brain [2]. Most of the cases of PD are idiopathic since their cause is unknown. Epigenetic factors that mediate mitochondrial dysfunction, inflammation, abrogation of the autosomal-lysosomal autophagy system and endoplasmic reticulum stress play a role in the progression of the disease [24]. It is a debilitating neurodegenerative disease characterized by bradykinesia and postural instability [31]. Loss of function/mutations in number of genes such as $\alpha$-synuclein, parkin, ubiquitin C-terminal hydrolase-1 (UCH-1), DJ-1, phosphatase and tensin homolog (PTEN) induced kinase 1 (PINK1), leucin-rich repeat kinase2 (LRRK2), omi/Htr A2, ATP13A2 and glucocerebrosidase accounts for the late onset of sporadic PD [32]. The over expression of mutant or

*Corresponding author; e-mail addresses: yasir_hasansiddique@rediffmail.com; yasirhasansiddique@gmail.com 
normal form of $\alpha$-synuclein lead to the formation of Lewy bodies which plays a crucial role in the pathogenesis of PD [4]. The formation of Lewy bodies has been linked with an increased oxidative stress in the brain of PD patients oxidative stress not only destroys the dopaminergic neurons but also compromises mitochondrial oxidative phosphorylation, leading to decreased energy output and eventually to secondary death of cells [2]. Despite of dopamine agonists and even L-dopamine treatment none yet address the underlying problem associated with it, i.e. the progressive loss of dopaminergic neurons [34]. Various epidemiological and biochemical studies have recently identified promising compounds that may elicit neuroprotection in PD [24, $28,29]$. Due to ethical reasons the experiments cannot be performed directly on human, hence model organisms such as mice, fruit flies and worms have been developed in order to understand the biology of the disease as well as pharmacological interventions [18]. A transgenic model of fruit flies expressing wild or mutant type of human alpha synuclein under GAL4/UAS system has been widely accepted [10]. In our earlier study the exposure to capsaicin has shown a delay in the loss of climbing ability in the same model of PD [26]. In the present study the effect of capsaicin was studied on the glutathione (GSH), protein carbonyl (PC) and dopamine contents, as well as the glutathione-S-transferase (GST) and monoamine oxidase (MAO) activities in the brain of PD model flies expressing human alpha synuclein.

\section{MATERIALS AND METHODS}

\section{Drosophila stocks}

Transgenic fly lines that express wild-type human synuclein (h- $\alpha \mathrm{S})$ under UAS control in neurons " $\left[\mathrm{w}\left[{ }^{*}\right] ; \mathrm{P}\{\mathrm{w}[+\mathrm{mC}]=\mathrm{UAS}-\mathrm{Hsap} / \mathrm{SNCA} . \mathrm{F}\} " 5 \mathrm{~B}\right.$ and GAL4 " $\mathrm{w}[*] ; \mathrm{P}\{\mathrm{w}[+\mathrm{mC}]=\mathrm{GAL} 4-$ elavL $\} " 3]$ were obtained from Bloomington Drosophila Stock Centre (Indiana University, Bloomington, IN). When the males of UAS (Upstream Activation Sequence)-Hsap/SNCA.F strains are crossed with the females of GAL4-elav. L (vice versa), the progeny expresses human $\alpha \mathrm{S}$ in the neurons [10].

\section{Drosophila culture and crosses}

The flies were cultured on standard Drosophila food containing agar, corn meal, sugar and yeast at $25^{\circ} \mathrm{C}(24 \pm 1)$ [30]. Crosses were set up as described in our earlier published work [27]. The PD flies were allowed to feed separately on different doses of capsaicin (Sigma, USA) mixed in the diet. Capsaicin was added to the diet at final concentration of 20, 40, 80 and $100 \mu \mathrm{M}$ for 24 days. The PD flies were also exposed to $10^{-3} \mathrm{M}$ of L-dopamine. The UASHsap/SNC.F act as a control. The control flies were allowed separately to feed on the selected doses of capsaicin. 


\section{Superoxide anion scavenging assay}

The inhibition of nitro blue tetrazolium (NBT) reduction by phenazin methosulphate (PMS) generated $\mathrm{O}_{2}{ }^{-}$- was used to determine the superoxide anion scavenging activity of the capsaicin [25]. The reaction mixture consisted of $75 \mu 1$ of each concentration of capsaicin, $750 \mu \mathrm{l}$ of Tris $\mathrm{HCl}(100 \mathrm{mM}$; pH 7.4); $187 \mu \mathrm{l}$ of NBT $(300 \mu \mathrm{M})$, $187 \mu \mathrm{l}$ of NADH $(936 \mu \mathrm{M})$. The reaction was initiated by adding phenazine methosulphate (PMS) $(120 \mu \mathrm{M})$. The reaction mixture was incubated at $25^{\circ} \mathrm{C}$ for $5 \mathrm{~min}$ and the OD was read at $560 \mathrm{~nm}$ and the degree of scavenging was calculated by the following equation:

$$
\begin{gathered}
\text { Scavenging }(\%)=\left[\frac{\mathrm{OD} \text { control }- \text { OD sample }}{\text { OD control }}\right] \times 100 \text {. } \\
\text { Diphenyl-picrylhydrazyl (DPPH) free radical scavenging }
\end{gathered}
$$

\section{Diphenyl-picrylhydrazyl (DPPH) free radical scavenging}

For estimating free radical scavenging potential of the capsaicin DPPH method as described by Wongsawatkul et al. [33] was used in the present study. When DPPH (a stable purple colour) react with an antioxidant, it is reduced to yield a light yellow coloured diphenyl picrylhydrazine. Color change was spectrophotometrically measured. The reaction mixture consisting of $500 \mu \mathrm{l}$ of capsaicin and $250 \mu \mathrm{l}$ of DPPH $(0.3 \mathrm{mM})$. The reaction mixture was shaken vigorously and allowed to stand at room temperature in the dark for $25 \mathrm{~min}$. The OD was read at $518 \mathrm{~nm}$ and the radical scavenging activity was calculated by the following equation:

$\%$ Radical scavenging $=(1-$ Absorbance of sample/Absorbance of control $) \times 100$.

\section{Preparation of homogenate}

Fly heads (50 heads) from each group (five replicates/group) were isolated and the homogenate was prepared in $0.1 \mathrm{M}$ phosphate buffer for the biochemical parameters.

\section{Estimation of glutathione content}

The glutathione (GSH) content was estimated colorimetrically using Ellman's reagent (DTNB) according to the procedure described by Jollow et al. [14]. The supernatant was precipitated with $4 \%$ sulphosalicyclic acid (4\%) in the ratio of $1: 1$. The samples were kept at $4{ }^{\circ} \mathrm{C}$ for $1 \mathrm{~h}$ and then subjected to centrifugation at $5000 \mathrm{rpm}$ for $10 \mathrm{~min}$ at $4{ }^{\circ} \mathrm{C}$. The assay mixture consisted of $550 \mu \mathrm{l}$ of $0.1 \mathrm{M}$ phosphate buffer, $100 \mu \mathrm{l}$ of supernatant and $100 \mu \mathrm{l}$ of DTNB. The OD was read at $412 \mathrm{~nm}$ and the results were expressed as $\mu$ moles of GSH/gram tissue. 


\section{Estimation of glutathione-S-transferase activity}

The glutathione-S-transferase (GST) activity was determined by the method of Habig et al. [11]. The reaction mixture consist of $500 \mu \mathrm{l}$ of $0.1 \mathrm{M}$ phosphate buffer, $150 \mu \mathrm{l}$ of $10 \mathrm{mM} \mathrm{CDNB}, 200 \mu \mathrm{l}$ of $10 \mathrm{mM}$ reduced glutathione and $50 \mu \mathrm{l}$ of supernatant. The OD was taken at $340 \mathrm{~nm}$ and the enzyme activity was expressed as $\mu$ moles of CDNB conjugates $/ \mathrm{min} / \mathrm{mg}$ protein.

\section{Lipid peroxidation assay}

Lipid peroxidation was measured according to the method described by Ohkawa et al. [19]. The reaction mixture consisted of $5 \mu \mathrm{l}$ of $10 \mathrm{mM}$ butyl-hydroxy toluene (BHT), $200 \mu \mathrm{l}$ of $0.67 \%$, thiobarbituric acid, $600 \mu \mathrm{l}$ of $1 \%$ O-phosphoric acid, $105 \mu 1$ of distilled water and $90 \mu \mathrm{l}$ of supernatant. The mixture was incubated at $90{ }^{\circ} \mathrm{C}$ for $45 \mathrm{~min}$ and the OD was measured at $535 \mathrm{~nm}$. The results were expressed as $\mu$ moles of TBARS formed/h/gram tissue.

\section{Estimation of protein carbonyl content (PCC)}

The PC content was estimated according to the protocol described by Hawkins et al. [12]. The brain homogenate was diluted to a protein concentration of approx. $1 \mathrm{mg} / \mathrm{ml}$. About $250 \mu \mathrm{l}$ of each diluted homogenate was taken in eppendorf centrifuge tubes separately. To it $250 \mu \mathrm{l}$ of $10 \mathrm{mM}$ 2,4-dinitrophenyl hydrazine (dissolved in $2.5 \mathrm{M} \mathrm{HCl}$ ) was added, vortexed and kept in dark for 20 min. About $125 \mu 1$ of 50\% $(\mathrm{w} / \mathrm{v})$ trichloroacetic acid (TCA) was added, mixed thoroughly and incubated at $-20{ }^{\circ} \mathrm{C}$ for $15 \mathrm{~min}$. The tubes were then centrifuged at $4{ }^{\circ} \mathrm{C}$ for $10 \mathrm{~min}$ at $9000 \mathrm{rpm}$. The supernatant was discarded and the pellet obtained was washed twice by ice cold ethanol: ethyl acetate (1:1). Finally, the pellets were re-dissolved in $1 \mathrm{ml}$ of $6 \mathrm{M}$ guanidine hydrochloride and the absorbance was read at $370 \mathrm{~nm}$.

\section{Estimation of monoamine oxidase (MAO)}

The method described by McEwen [17] was used to estimate the monoamine oxidase activity. The assay mixture consisted of $400 \mu \mathrm{L}$ of $0.1 \mathrm{M}$ phosphate buffer $(\mathrm{pH} 7.4)$, $1300 \mu \mathrm{L}$ of distilled water, $100 \mu \mathrm{L}$ of benzylamine hydrochloride and $200 \mu \mathrm{L}$ of brain homogenate. The assay mixture was incubated for $30 \mathrm{~min}$ at room temperature and then $1 \mathrm{~mL}$ of $10 \%$ perchloric acid was added and centrifuged at $1500 \mathrm{~g}$ for $10 \mathrm{~min}$. The OD was taken at $280 \mathrm{~nm}$. 


\section{Dopamine content determination}

Dopamine content was measured by the method described by Schlumpf et al. [23] Fifty heads of flies (5 replicates/group) from each group were taken in $500 \mu \mathrm{l}$ of $\mathrm{HCl}$ butanol $(0.85 \mathrm{ml}$ of $37 \% \mathrm{HCl}$ in 1 litre $n$-butanol). After homogenization the samples were centrifuged at $3000 \mathrm{rpm}$ for $5 \mathrm{~min}$. After collecting the supernatant, $250 \mu \mathrm{l}$ of heptane and $100 \mu \mathrm{l}$ of $0.1 \mathrm{M} \mathrm{HCl}$ were added. The samples were vortexed and centrifuged at $3000 \mathrm{rpm}$ for $5 \mathrm{~min}$. The upper organic phase was discarded and the lower aqueous phase was kept for dopamine assay. To $100 \mu 1$ of aqueous phase, $50 \mu 10.4 \mathrm{M}$ $\mathrm{HCl}, 100 \mu \mathrm{l}$ of sodium acetate buffer ( $\mathrm{pH}$ 6.9), $100 \mu \mathrm{l}$ of iodine solution was added and kept for two min. The reaction was stopped by adding of $100 \mu \mathrm{l}$ of sodium sulphite solution. After two min, $100 \mu \mathrm{l}$ of acetic acid (10M) was added and then the mixture was heated at $100^{\circ} \mathrm{C}$ for $6 \mathrm{~min}$. The OD was taken at $375 \mathrm{~nm}$ after cooling the samples at room temperature.

\section{Statistical analysis}

The statistical analysis was done by using one-way ANOVA.

\section{RESULTS AND DISCUSSION}

A dose dependent significant increase in the potential of scavenging superoxide anion (Fig. 1A) as well as free radicals (Fig. 1B) was observed for each of the selected doses of capsaicin. Oxidative stress is a major factor for the pathogenesis of PD, especially due to the death of dopaminergic neurons [20]. Natural plant products have a variety of biological activities and also have potential to influence pathogenesis of neurodegenerative disease [16]. The mechanisms responsible for neurodegeneration in PD are largely unknown, although researches have suggested that mitochondrial complex dysfunction, oxidative stress and abrupt proteolytic degradation may also contribute to the pathogenesis of the disease [32]. GSH is the most abundant thiols present in the

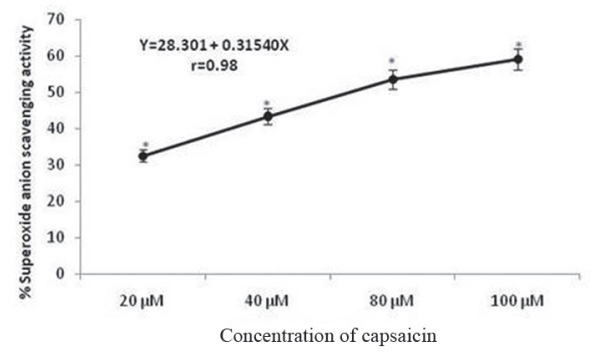

(A)

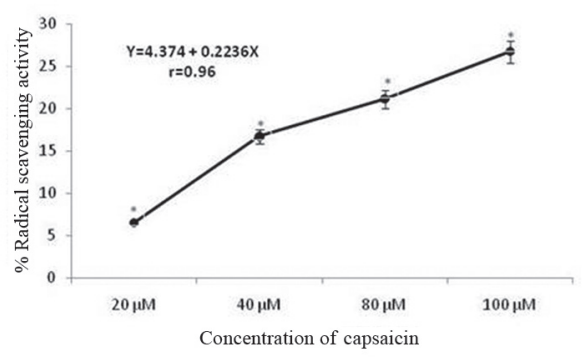

(B)

Fig. 1. (A) Superoxide anion scavenging activity of capsaicin and (B) Radical scavenging activity of capsaicin. The values are the mean of five assays [* significant with respect to control, $p<0.05$ ] 

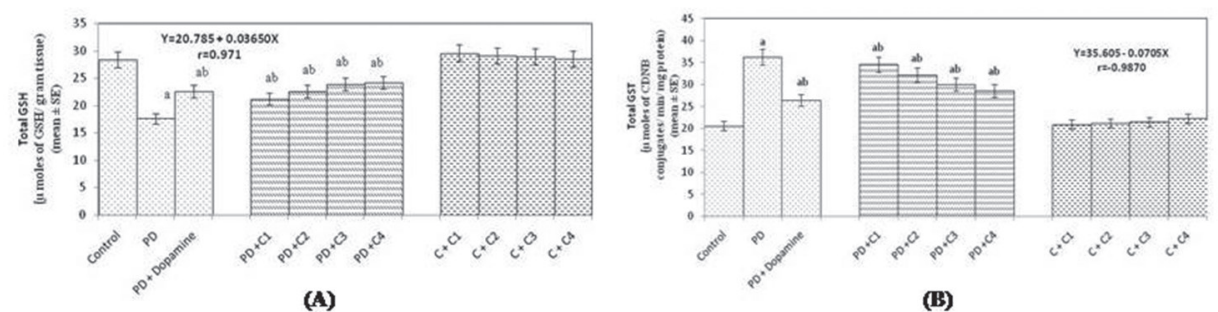

(B)

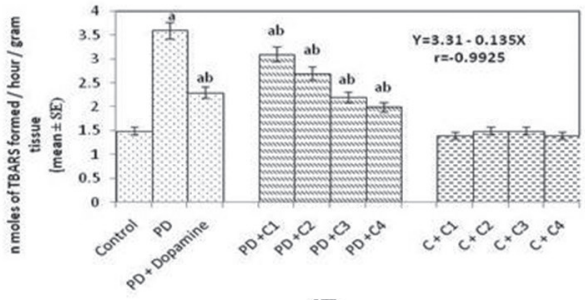

(C)

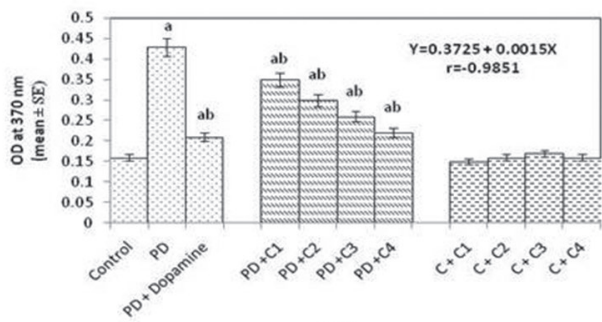

(D)

Fig. 2. (A) Effect of capsaicin on the glutathione (GSH), (B) glutathione-S-transferase (GST) activity, (C) Lipid peroxidation and (D) Protein carbonyl content in the brains of flies $[\mathrm{C} 1=20 \mu \mathrm{M}$; $22=40 \mu \mathrm{M}$; $\mathrm{C} 3=80 \mu \mathrm{M} ; \mathrm{C} 4=100 \mu \mathrm{M} ; \mathrm{PD}=\mathrm{PD}$ flies; Dopamine $=10^{-3} \mathrm{M} ; \mathrm{N}=50$ heads]. The flies were allowed to feed on the diet supplemented with capsaicin for 24 days and then assayed for GSH content. [ ${ }^{a}$ significant difference with respect to control, $p<0.05$; 'bignificant difference with respect to PD flies $p<0.05$ ]

eukaryotic cells. The depletion in the content of GSH is an indication of cytotoxicity [13]. The results obtained for the GSH content showed a significant 1.61-fold of decrease in the GSH content (Fig. 2A; $p<0.05$ ). The exposure of PD flies to 20, 40, 80 and $100 \mu \mathrm{M}$ of capsaicin showed a dose dependent significant increase of 1.20-, 1.28-, 1.35-, 1.37-folds in the GSH content compared to unexposed PD flies (Fig. 2A; $p<0.05, r=0.971)$. GSTs are enzymes responsible for the detoxification and hence an increased GST activity indicates a state of oxidative stress [15]. The GST activity showed a significant increase of 1.76-fold compared to control flies (Fig. 2B; $p<0.05$ ). The exposure of PD flies to $20,40,80$ and $100 \mu \mathrm{M}$ of capsaicin, respectively, showed a dose dependent significant decrease of 1.04-, 1.12-, 1.20-, 1.26-folds, respectively in GST activity (Fig. 2B; $p<0.05, r=-0.987$ ). Glutathione, a tripeptide, is responsible for hydrophilic xenobiotics conjugation. The sulphydryl group is essential for its antioxidant activity against some forms of reactive oxygen species (ROS) in cells [9]. There are evidences that natural plant products can increase the intracellular basal level of GSH in order to combat the generation of free radicals [6]. The generated free radicals can attack unsaturated lipids in a cell, resulting in a chain reaction of the formation of free radicals [9]. The end products of this reaction are lipid alcohols, aldehydes and malondialdehyde (MDA). Therefore the measurement of the MDA is a common method for the toxicological evaluation [9]. The PD flies showed an increase of 2.4-fold in the lipid peroxidation compared to control flies (Fig. 2C; $p<0.005$ ). The PD flies exposed to $20,40,80$ and $100 \mu \mathrm{M}$ of capsaicin 
showed a dose dependent significant decrease of 1.16-, 1.33-, 1.63-, 1.8-folds in lipid peroxidation compared to unexposed PD flies (Fig. 2C; $p<0.05, r=-0.9925$ ). The PD flies showed a significant increase of 2.68 -fold in the PC content compared to control flies (Fig. 2D; $p<0.05$ ). The PD flies exposed to 20, 40, 80 and $100 \mu \mathrm{M}$ of capsaicin, respectively, showed a dose dependent significant decrease in the PC content of 1.22-, 1.43-, 1.65-, 1.95-folds compared to unexposed PD flies (Fig. 2D; $p<0.05, r=-0.9851$ ). In our present study the PD flies showed an increase in LPO, PC content, GST activity and decrease in GSH content, however, the exposure of PD flies to capsaicin resulted in a decrease in LPO, PC content, GST activity and increase in GSH content in a dose dependent manner. Reactive intermediates can react with GSH either by a direct chemical reaction or by a GST mediated reaction preventing the possible cell death. Capsaicin has been reported to scavenge free radicals and reduced the lipid peroxidation in rat brain homogenate thus proving its implication in the prevention or treatment of neurodegenerative disease [7]. It also reduced Alzheimer associated tau changes in the hippocampus of type 2 diabetes rats [35]. In our study the PD flies showed an increase levels of MAO but flies exposed to capsaicin showed a decrease in the activity MAO in a dose dependent manner. The PD flies showed a significant increase of 4.42 -fold in the activity of MAO (Fig. 3A; $p<0.05$ ). The PD flies exposed to $20,40,80$ and $100 \mu \mathrm{M}$ of capsaicin, respectively, showed a dose dependent significant decrease of 1.09-, 1.24-, 1.47-, 1.60-folds in MAO activity, compared to unexposed PD flies (Fig. 3A; $p<0.05, r=0.991$ ). MAO is an iron containing enzyme that has been reported to maintain the neuron firing rate and is also involved in the metabolism of dopamine [8]. Our results obtained on superoxide anion and free radical scavenging assays performed on the selected doses of capsaicin showed that it has a potential of scavenging free radicals and superoxide anions. Hence, its antioxidant potential may contribute to recover the impaired functions of PD flies. The balance between oxidation and anti-oxidation is believed to be critical in maintaining healthy biological systems. Under normal conditions, the antioxidant defense system allows the elimination of excess ROS, including superoxide anions and free radicals [3].

Our present findings also suggest an increase in the dopamine content in the brain of PD flies exposed to capsaicin. The PD flies showed a significant decrease of 2.08fold in the dopamine content, compared to control flies (Fig. 3B; $p<0.05$ ). The PD flies exposed to $20,40,80$ and $100 \mu \mathrm{M}$ of capsaicin showed a dose dependent significant increase of 1.23-, 1.39-, 1.51-, 1.56-folds in the dopamine content compared to unexposed PD flies (Fig. 3B; $p<0.05, r=0.97$ ). Pathologically, PD is characterized by the degeneration of dopaminergic neurons in the substantia nigra pars compacta that leads to the depletion of dopamine [21]. In our study the exposure of PD flies to capsaicin leads to an increase of the dopamine content. Capsaicin has been reported to prevent the degeneration of dopamine neurons by inhibiting glial activation and oxidative stress in the 1-methyl-4-phenyl-1,2,3,6-tetrahydropyridine (MPTP) mouse model of PD [5]. Our earlier studies on the PD model flies demonstrated that the exposure of flavonoid protects the dopaminergic neurons [1], and the study with geraniol on mice also suggests that the natural plant products are effective in protect- 
ing the dopaminergic neurons and thereby results in maintaining the appropriate levels of dopamine [22]. However, our earlier study with geraniol showed that it does not alter the expression of $\alpha$-synuclein and the formation of Lewy bodies, but its antioxidant potential is responsible for delaying the PD symptoms in the PD model flies [29]. It is concluded that capsaicin is potent in recovering the impaired functions of PD flies.

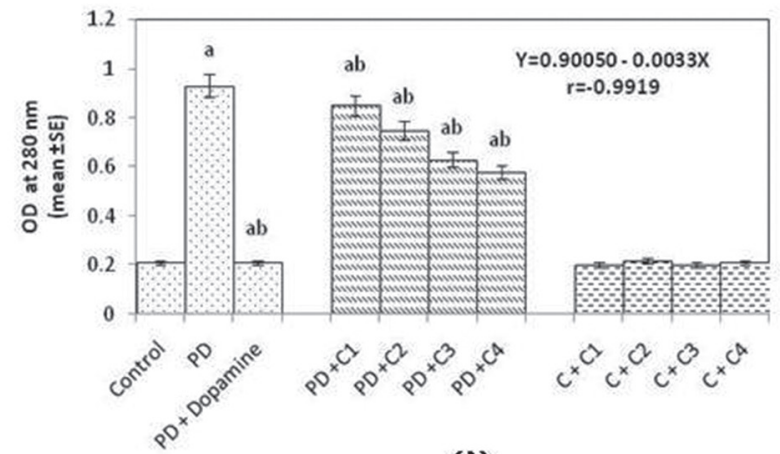

(A)

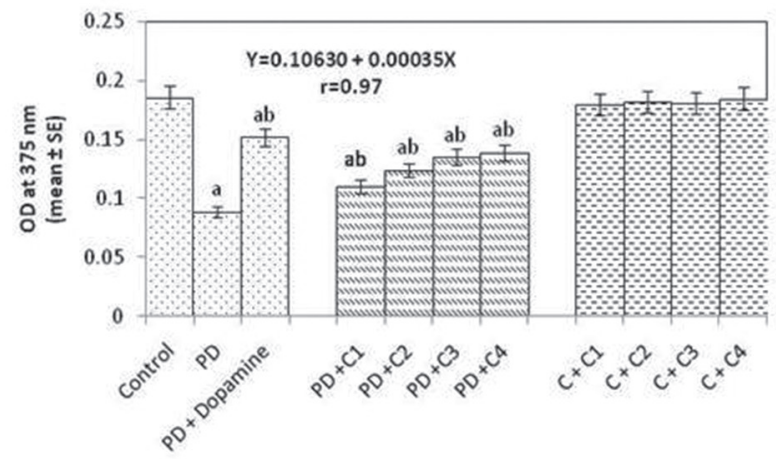

Fig. 3. (A) Effect of capsaicin on the monoamine oxidase activity and (B) Dopamine content measured in the brains of flies $[\mathrm{C} 1=20 \mu \mathrm{M} ; \mathrm{C} 2=40 \mu \mathrm{M} ; \mathrm{C} 3=80 \mu \mathrm{M} ; \mathrm{C} 4=100 \mu \mathrm{M} ; \quad \mathrm{PD}=\mathrm{PD}$ flies; Dopamine $=10^{-3} \mathrm{M} ; \mathrm{N}=50$ heads]. The flies were allowed to feed on the diet supplemented with capsaicin for 24 days and then assayed for monoamine oxidase activity. [asignificant difference with respect to control, $p<0.05$; ${ }^{b}$ significant difference with respect to PD flies $p<0.05$ ]

\section{ACKNOWLEDGEMENTS}

The Grant (No. 59/58/2011/BMS-TRM) received from Indian Council of Medical Research (ICMR), New Delhi to the author YHS is thankfully acknowledged. The flies for the experiments were purchased from Bloomington Drosophila Stock Centre, Department of Biology, Indiana University, Bloomington, IN, USA. We are also thankful to the Chairman, Department of Zoology, AMU, Aligarh for providing the lab facility. 


\section{REFERENCES}

1. Ara, G., Afzal, M., Jyoti, S., Siddique, Y. H. (2017) Effect of myricetin on the oxidative stress markers in the brain of transgenic flies expressing human alpha synuclein. Int. J. Nut. Pharmacol. Neuro. Dis. 7, 101-106.

2. Baluchnejadmojarad, T., Roghani, M., Nadoushan, M. R., Bagheri, M. (2009) Neuroprotective effect of genistein in 6-hydroxydopamine hemi-parkinsonian rat model. Phytotherap. Res. 23, 132-135.

3. Bouayed, J., Bohn, T. (2010) Exogenous antioxidants-double-edged swords in cellular redox state: health beneficial effects at physiologic doses versus deleterious effects at high doses. Oxi. Med. Cell long. 3, 228-237.

4. Breda, C., Giorgini, F., Steinert, J. R. (2015) Synapses and $\alpha$-synuclein signalling in disease. Cogent Biol. 1, 1085295.

5. Chung, Y. C., Baek, J. Y., Kim, S. R., Ko, H.W., Bok, E., Shin, W. H., Jin, B. K. (2017) Capsaicin prevents degeneration of dopamine neurons by inhibiting glial activation and oxidative stress in the MPTP model of Parkinson's disease. Exp. Mol. Med. 49, e298.

6. Cipak, L., Berczeliová, E., Paulikova, H. (2003) Effects of flavonoids on glutathione and glutathionerelated enzymes in cisplatin-treated L1210 leukemia cells. Neoplasma 50, 443-446.

7. Dairam, A., Fogel, R., Daya, S., Limson, J. L. (2008) Antioxidant and iron-binding properties of curcumin, capsaicin, and S-allylcysteine reduce oxidative stress in rat brain homogenate. J. Agr. Food Chem. 56, 3350-3356.

8. Dreiseitel, A., Korte, G., Schreier, P., Oehme, A., Locher, S., Domani, M., Sand, P. G. (2009) Berry anthocyanins and their aglycons inhibit monoamine oxidases A and B. Pharmacol. Res. 59, 306-311.

9. Durgo, K., Vuković, L., Rusak, G., Osmak, M., Franekić, Čolić, J. (2007) Effect of flavonoids on glutathione level, lipid peroxidation and cytochrome P450 CYP1A1 expression in human laryngeal carcinoma cell lines. Food Tech. Biotech. 45, 69-79.

10. Feany, M. B., Bender, W. W. (2000) A Drosophila model of Parkinson's disease. Nature 404, 394 398.

11. Habig, W. H., Pabst, M. J., Fleischner, G., Gatmaitan, Z., Arias, I. M., Jakoby, W. B. (1974) The identity of glutathione S-transferase B with ligandin, a major binding protein of liver. Proc. Natl Acad. Sci. 71, 3879-3882.

12. Hawkins, C. L., Morgan, P. E., Davies, M. J. (2009) Quantification of protein modification by oxidants. Free Radic. Biol. Med. 46, 965-988.

13. Jeng, J. H., Tsai, C. L., Hahn, L. J., Yang, P. J., Kuo, Y. S., Kuo, M. Y. P. (1999) Arecoline cytotoxicity on human oral mucosal fibroblasts related to cellular thiol and esterase activities. Food Chem Toxicol. 37, 751-756.

14. Jollow, D. J., Mitchell, J. R., Zampaglione, N. A., Gillette, J. R. (1974) Bromobenzene-induced liver necrosis. Protective role of glutathione and evidence for 3,4-bromobenzene oxide as the hepatotoxic metabolite. Pharmacology 11, 151-169.

15. Kölsch, H., Linnebank, M., Lütjohann, D., Jessen, F., Wüllner, U., Harbrecht, U., Von Bergmann, K. (2004) Polymorphisms in glutathione S-transferase omega-1 and AD, vascular dementia, and stroke. Neurology 63, 2255-2260.

16. Liu, Y., Gou, L. S., Tian, X., Fu, X. B., Ling, X., Sun, L. Y., Yin, X. X. (2013) Protective effects of luteolin on cognitive impairments induced by psychological stress in mice. Exp. Biol. Med. 238, 418-425.

17. McEwen, C. M. (1965) Human plasma monoamine oxidase I. Purification and identification. J. Biol. Chem. 240, 2003-2010.

18. Munoz-Soriano, V., Paricio, N. (2011) Drosophila models of Parkinson's disease: discovering relevant pathways and novel therapeutic strategies. Parkinson's Dis. 2011, 1-14.

19. Ohkawa, H., Ohishi, N., Yagi, K. (1978) Reaction of linoleic acid hydroperoxide with thiobarbituric acid. J. Lipid Res. 19, 1053-1057. 
20. Olanow, C. W., Tatton, W. G. (1999) Etiology and pathogenesis of Parkinson's disease. Ann. Rev Neurosci. 22, 123-144.

21. Recchia, A., Debetto, P., Negro, A., Guidolin, D., Skaper, S. D., Giusti, P. (2004) $\alpha$-Synuclein and Parkinson's disease. The FASEB J. 18, 617-626.

22. Rekha, K. R., Selvakumar, G. P., Sethupathy, S., Santha, K., Sivakamasundari, R. I. (2013) Geraniol ameliorates the motor behavior and neurotrophic factors inadequacy in MPTP-induced mice model of Parkinson's disease. J. Mol. Neurosci. 51, 851-862.

23. Schlumpf, M., Lichtensteiger, W., Langemann, H., Waser, P. G., Hefti, F. (1974) A fluorometric micromethod forthe simultaneous determination of serotonin, noradrenaline and dopamine in milligram amounts of brain tissue. Biochem. Pharmacol. 23, 2437-2446.

24. Seidl, S. E., Santiago, J. A., Bilyk, H., Potashkin, J. A. (2014) The emerging role of nutrition in Parkinson's disease. Front Aging Neurosci. 6, 36.

25. Sghaier, M. B., Bhouri, W., Neffati, A., Boubaker, J., Skandrani, I., Bouhlel, I., Ghedira, K. (2011) Chemical investigation of different crude extracts from Teucrium ramosissimum leaves. Correlation with their antigenotoxic and antioxidant properties. Food Chem. Toxicol. 49, 191-201.

26. Siddique, Y. H., Ara, G., Jyoti, S., Afzal, M. (2012) Effect of capsaicin on the climbing ability in Drosophila model of Parkinson's disease. Am. J. Drug Dis. Dev. 2, 50-54.

27. Siddique, Y. H., Fatima, A., Jyoti, S., Naz, F., Rahul, Khan, W., Singh, B. R., Naqvi, A. H. (2015) Evaluation of the toxic potential of graphene copper nanocomposite (GCNC) in the third instar larvae of transgenic Drosophila melanogaster (hsp70-lacZ)Bg9. PLOS one 8, e80944.

28. Siddique, Y. H., Jyoti, S. (2017) Alteration in biochemical parameters in the brain of transgenic Drosophila melanogaster model of Parkinson's disease exposed to apigenin. Int. Med. Res. 6, 245253.

29. Siddique, Y. H., Naz, F., Jyoti, S., Ali, F., Fatima, A. (2016) Protective effect of Geraniol on the transgenic Drosophila model of Parkinson's disease. Environ. Toxicol Pharmacol. 43, 225-231.

30. Siddique, Y. H., Naz, F., Jyoti, S. (2014) Effect of curcumin on lifespan, activity pattern, oxidative stress, and apoptosis in the brains of transgenic Drosophila model of Parkinson's disease. BioMed Res. Int. 2014, 1-6.

31. Tan, L. C., Koh, W. P., Yuan, J. M., Wang, R., Au, W. L., Tan, J. H., Yu, M. C. (2007) Differential effects of black versus green tea on risk of Parkinson's disease in the Singapore Chinese Health Study. Am. J. Epidem. 167, 553-560.

32. Whitworth, A. J., Theodore, D. A., Greene, J. C., Beneš, H., Wes, P. D., Pallanck, L. J. (2005) Increased glutathione S-transferase activity rescues dopaminergic neuron loss in a Drosophila model of Parkinson's disease. Proc. Natl Acad. Sci. 102, 8024-8029.

33. Wongsawatkul, O., Prachayasittikul, S., Isarankura-Na-Ayudhya, C., Satayavivad, J., Ruchirawat, S. Prachayasittikul, V. (2008) Vasorelaxant and antioxidant activities of Spilanthes acmella Murr. Int. J. Mol. Sci. 9, 2724-2744.

34. Wu, S. S., Frucht, S. J. (2005) Treatment of Parkinson's disease. CNS Drugs 19, 723-743.

35. Xu, W., Liu, J., Ma, D., Yuan, G., Lu, Y., Yang, Y. (2017) Capsaicin reduces Alzheimer-associated tau changes in the hippocampus of type 2 diabetes rats. PloS one 12, e0172477. 